\title{
Numismatic Image Segmentation: An Empirical Study
}

\author{
Anindya Halder, PhD \\ Dept. of Computer Application \\ North-Eastern Hill University \\ Tura Campus, Meghalaya
}

\author{
Amit Kumar Upadhyay, $\mathrm{PhD}$ \\ Dept. of History \& Archeology \\ North-Eastern Hill University \\ Tura Campus, Meghalaya
}

\author{
Sujit Kumar Das \\ Dept. of Computer Application \\ North-Eastern Hill University \\ Tura Campus, Meghalaya
}

\begin{abstract}
Numismatic coins are one of the important elements to know history of economic, culture and society in ancient as well as present time. Extraction of information from numismatic coins in traditional manual method sometimes contains human error and in the same time it is time consuming. Engineering in this aspects deal with problems of traditional method. Engineering tasks namely pattern recognition, computer vision, and object authentication are high level computational tasks where machine deal with image data for processing. To do these high level tasks image segmentation is a basic process. Its purpose is to separate the targets from the background in an image in order to provide the basis for the subsequent sorting, recognition and indexing. Again segmentation methods depends on types of image data, we are dealing with. In this paper we discussed some existing methods of image segmentation and tried to find out suitable method for coin images. Maximum Entropy Thresholding(MET) based on normalised histogram method giving us more suitable segmented image.
\end{abstract}

\section{General Terms}

Numismatic data processing, Segmentation, Image Processing, Pattern Recognition

\section{Keywords}

Numismatic data, Hough Transformation, Maximum Entropy Thresholding

\section{INTRODUCTION}

To reconstruct our past numismatic study is one of the most important fields. By the phrase "Numismatic Coins" we mean ancient coins which prove its own importance in History where the other sources (e.g. Inscriptions, literature) of study are even silent. Although coins are small in size, they contains lots of information our present as well as ancient society, their economy, culture and religion etc .According to historian two types of study of coins are internal which contains information like techniques used in making of any coin, about metal and mixture of different material, value etc. And other one is external which deals with the study and analysis of different legends, symbols and mint marks, etc. In case of external study of the coins, the decipherment of the legends, symbols, and mint marks of the coins is very important for proper understanding of the political and socio-economic situation, the religion and cultural heritage of the past society. The traditional methods for external study of deciphering any coin are very manual and limited in nature and require lots of professionalism as in the manual method there is always a chance of having human error. Therefore, the final decipherment results produced by traditional manual methods are often not unanimously acceptable among the Historians.

Computer vision comes into existence to deal with traditional problems. Again for any high level computational tasks like pattern recognition, coin recognition image segmentation in the initial phase of processing plays an important role. In segmentation, value is assigned to every pixel of an image in such a way that the pixels which share certain characteristics, such as colour, intensity or texture in a particular region are grouped together. Adjacent regions that are not grouped together must be significantly different with respect to the same characteristics. Purpose of dividing an image is to further analyze each of these subparts or sub-images so that some high level information can be extracted. Sometimes image de-noising is done for the segmentation to enhance the image and improve the quality of segmentation process [5].

\section{NUMISMATIC DATA}

We took data from "American Numismatic Society" for our experiments. The ANS collections database contains information on more than 600,000 objects in the Society's collections. These include coins, paper money, tokens, 'primitive' money, medals and decorations, from all parts of the world, and all periods in which such objects have been produced. Our main focus is on coins data only. We have collected digital image of numismatic coins with their details as given in the database. The summary of the numismatic data are shown in Table 1 below.

\begin{tabular}{|c|l|l|}
\hline \multicolumn{2}{|c|}{ Table 1. Summary of the numismatic data } \\
\hline Collectio Name & Digital Image & \multicolumn{1}{|c|}{ Details of Data } \\
\hline Byzantine & $\begin{array}{l}\text { It comprises some 13,000 coins } \\
\text { struck at Byzantium and the } \\
\text { regional mints of the Byzantine } \\
\text { Empire from the reign of } \\
\text { Anastasius I (AD 419-518). }\end{array}$ \\
\hline East Asian & $\begin{array}{l}\text { The Department of East Asian } \\
\text { Coins comprises some 50,000 } \\
\text { coins and other objects produced } \\
\text { in the area of modern China, } \\
\text { Korea, Japan, and Vietnam. The } \\
\text { Department includes all coins } \\
\text { struck in these regions from the } \\
\text { beginning of coinage in the 6th } \\
\text { century BC down to the modern } \\
\text { day. It also includes paper money }\end{array}$ \\
\hline Greek & $\begin{array}{l}\text { The collection of ancient Greek } \\
\text { coins comprises some 100,000 } \\
\text { items, classified according to } \\
\text { regions and mints of the ancient } \\
\text { world, from Spain and North } \\
\text { Africa. In the west to Afghanistan } \\
\text { in the east. It includes all Greek } \\
\text { coins. }\end{array}$ \\
\hline
\end{tabular}




\begin{tabular}{|c|l|l|}
\hline Islamic & $\begin{array}{l}\text { The Islamic Department contains } \\
\text { over 70,000 coins and other } \\
\text { objects. It includes all coins and } \\
\text { paper money from North Africa. }\end{array}$ \\
\hline $\begin{array}{c}\text { Latin } \\
\text { American }\end{array}$ & $\begin{array}{l}\text { The Latin American Department } \\
\text { contains coins and paper money of } \\
\text { Central America, South American } \\
\text { and the Caribbean. The total figure } \\
\text { for the Latin American cabinet is } \\
\text { approximately 30,000 pieces. }\end{array}$ \\
\hline Medal and & $\begin{array}{l}\text { It contains more than 50,000 } \\
\text { medals from around the world of } \\
\text { all varieties, including } \\
\text { commemorative medals, art } \\
\text { medals and society medals. }\end{array}$ \\
\hline Modern & $\begin{array}{l}\text { The Modern Department includes } \\
\text { all coins minted by minting } \\
\text { machinery and all paper money } \\
\text { produced in Europe, Canada, } \\
\text { Oceania and sub-Saharan Africa. } \\
\text { The total figure for the modern } \\
\text { cabinet is approximately 100,000 } \\
\text { pieces. }\end{array}$ \\
\hline
\end{tabular}

\section{RELATED WORK}

Coin segmentation is the extraction of the coin region from the photo. Based on ideal assumptions of coin photos, two methods are usually used to segment round coins: the global thresholding applied by applied by N"olle, et al. [1] and Van Der Maaten, et al. [2] and the Hough transform for circle detection applied by Reisert, et al. [3]. To segment non-round ancient coins, Zambanini and Kampel [4] proposed a relatively sophisticated approach: they apply seven discrete thresholds on the intensity image obtained by local entropy and local range of gray values, and then select the best segmented region based on a confidence score computed by the shape factor of circularity. However, all stated methods are developed only for round or almost round coins.

\subsection{Global Thresholding}

Global (single) thresholding[7]method is used when there the intensity distribution between the objects of foreground and background are very distinct. When the difference between foreground and background objects is very distinct, a single value of threshold can simply be used to differentiate both objects apart. Thus, in this type of thresholding, the value of threshold $\mathrm{T}$ depends solely on the property of the pixel and the grey level value of the image [6].

\section{Automatic Threshold Selection Algorithm}

1. An initial threshold (T) is chosen; this can be done randomly or according to any other method desired.

2. The image is segmented into object and background pixels as described above, creating two sets:

$$
\begin{array}{ll}
\text { i. } & \mathrm{G} 1=\{\mathrm{f}(\mathrm{m}, \mathrm{n}): \mathrm{f}(\mathrm{m}, \mathrm{n})>\mathrm{T}\} \text { (object pixels) } \\
\text { i. } & \mathrm{G} 2=\{\mathrm{f}(\mathrm{m}, \mathrm{n}): \mathrm{f}(\mathrm{m}, \mathrm{n})<=\mathrm{T}\} \text { (background pixels) }
\end{array}
$$

3. The average of each set is computed

$$
\begin{aligned}
& \text { i. } \mathrm{M} 1=\text { average value of } \mathrm{G} 1 \\
& \text { i. } \mathrm{M} 2=\text { average value of } \mathrm{G} 2 .
\end{aligned}
$$

4. A new threshold is created that is the average of M1 and M2.

$$
\mathrm{T}^{\prime}=(\mathrm{M} 1+\mathrm{M} 2) / 2
$$

5. Go back to step two, now using the new threshold computed in step four, keep repeating until the new threshold matches the one before it (i.e., until convergence has been reached).

\subsection{Hough Transformation}

The Hough transform is a feature extraction technique used in image analysis, computer vision, and digital image processing. The purpose of the technique is to find imperfect instances of objects within a certain class of shapes by a voting procedure. This voting procedure is carried out in a parameter space, from which object candidates are obtained as local maxima in a so-called accumulator space that is explicitly constructed by the algorithm for computing the Hough transform.

This Hough transform is highly optimized. It uses the midpoint circle algorithm to draw the circles in voting space quickly and without gaps. It also includes an option for searching only part of the image to increase speed if a rough estimate of the circle locations is known.

\section{Midpoint Circle Algorithm:}

It is very similar to Bresenhams approach. It is based on the following functions for testing the spatial relationship between an arbitrary point $(\mathrm{x}, \mathrm{y})$ and a circle of radius centered at the origin.

$$
\begin{aligned}
& F(x, y)=X^{2}+Y^{2}+r^{2} \\
& F(x, y) \text { will be } 0 \text { if }(x, y) \text { on the circle. } \\
& \text { Will be }>0 \text { if }(x, y) \text { outside. } \\
& \text { Will be }<0 \text { if }(x, y) \text { inside. }
\end{aligned}
$$

Now consider the coordinates of the point halfway between pixel $T \& S\left(X_{i}+1, Y_{i}-1 / 2\right)$. This is called midpoint and we use it to define a decision parameter:

$$
P_{i}=f\left(X_{i}+1, Y_{i}-1\right)=\left(X_{i}+1\right)^{2}+\left(Y_{i}-1 / 2\right)^{2}-r^{2}
$$

If $\mathrm{Pi}-\mathrm{ve}$, the midpoint is inside the circle, then choose pixel $\mathrm{T}$. If $\mathrm{Pi}$ is $+v e$, the midpoint is outside the circle and choose pixel $\mathrm{S}$.

Similarly, the decision parameter for the next step is:

$P_{i+1}=\left(X_{i+1}+1\right)^{2}+\left(Y_{i+1}-1 / 2\right)^{2}-r^{2}$

Since $X_{i+1}=X_{i+1} P_{i+1}-P_{i}=\left[\left(X_{i+1}\right)+1\right]^{2}-\left(X_{i+1}\right)^{2}+\left(Y_{i+1}-\right.$ $1 / 2)^{2}-\left(Y_{i}-1 / 2\right)^{2}$

Hence $\mathrm{P}_{\mathrm{i}+1}=\mathrm{P}_{\mathrm{i}}+2\left(\mathrm{X}_{\mathrm{i}}+1\right)+1+\left(\mathrm{Y}_{\mathrm{i}+1}^{2}-\mathrm{Y}_{\mathrm{i}}^{2}\right)-\left(\mathrm{Y}_{\mathrm{i}+1}-\mathrm{Y}_{\mathrm{i}}\right)$

If pixel $\mathrm{T}$ is chosen (meaning $\mathrm{Pi}<0$ ), we have $\mathrm{Y}_{\mathrm{i}+1}=\mathrm{Y}_{\mathrm{i}}$

If pixel $\mathrm{S}$ is chosen (meaning $\mathrm{Pi}>0$ ), we have $\mathrm{Y}_{\mathrm{i}+1}=\mathrm{Y}_{\mathrm{i}-1}$. Thus, $\mathrm{P}_{\mathrm{i}+1}=\mathrm{Pi}+2\left(\mathrm{X}_{\mathrm{i}+1}\right)+1$ if $\mathrm{Pi}<0 \mathrm{Pi}+2\left(\mathrm{X}_{\mathrm{i}+1}\right)+1-2\left(\mathrm{Y}_{\mathrm{i}-1}\right)$ if $\mathrm{Pi}>0$. In terms of $(\mathrm{Xi}, \mathrm{Yi})$, we have $\mathrm{P}_{\mathrm{i}+1}=\mathrm{Pi}+2 \mathrm{X}_{\mathrm{i}}+3$ if $\mathrm{P}_{\mathrm{i}}<0 \mathrm{P}_{\mathrm{i}}+2(\mathrm{Xi}-\mathrm{Yi})+5$ if $\mathrm{P}_{\mathrm{i}}>0$

Finally, compute the initial value for the decision parameter using the original definition of pi and $(0, \mathrm{r}): \mathrm{Pi}=(0+1)^{2}+(\mathrm{r}-$ $1 / 2)^{2}-r^{2}=5 / 4-r$ One can see that this is not really integer 
computation. However, when $r$ is an integer we can simply set $P_{1}=1-r$. The error of being $1 / 4$ less than the precise value does not prevent $P_{1}$ from getting the appropriate sign.

It does not affect the rest of the scan conversion process, because the decision variable is only updated with integer increment in subsequent steps.

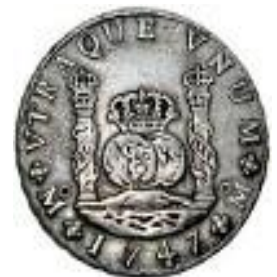

Fig 1: (a) Input Image

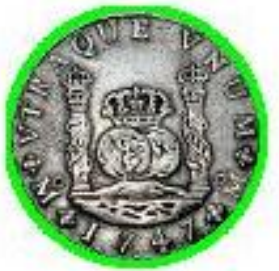

(b)Transformed Image
3.3 Numismatic Coin Segmentation based on MET In information theory, entropy is used to quantify the amount of information the entropy reflects the information content of symbols independent of any particular probability model. Image analysis takes the concept of entropy in the sense of information theory (Shannon entropy), where entropy is used to quantify the minimum descriptive complexity of a random variable. Because the entropy can provide a good level of

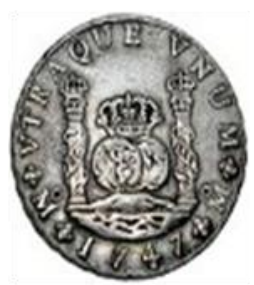

Fig 2: (a) Latin american

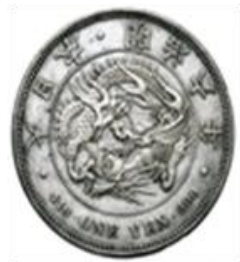

Fig 3: (a) East Asian

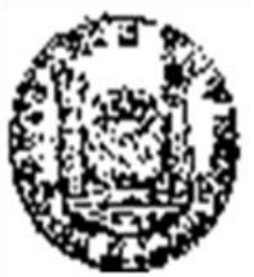

(b) Global Thresholding

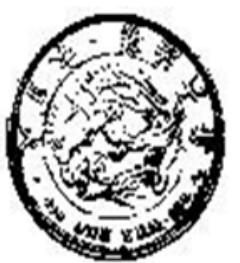

(b) Global Thresholding

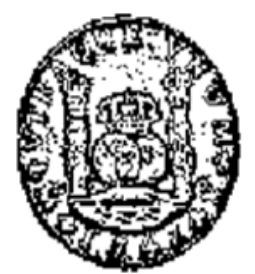

(c) Adaptive Thresholding

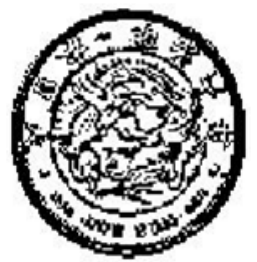

(c) Adaptive Thresholding

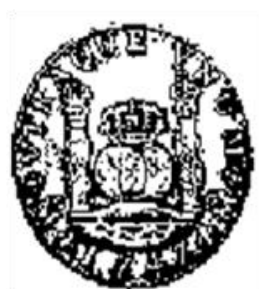

(d) MET information to describe a given image, we can compute the entropy of the distribution of gray levels and obtain an appropriate partition for target image.

Maximum Entropy Thresholding (MET) is the maximization Let $\mathrm{C}_{1}$ and $\mathrm{C}_{2}$ two classes for the object and the background respectively; the maximum entropy measure can be calculated

$$
\begin{aligned}
& \mathrm{hC}_{1}(\mathrm{t})=\operatorname{sum}\left(\mathrm{pi} / \mathrm{pC}_{1}\right) * \log \left(\mathrm{pi} / \mathrm{pC}_{1}\right) \text { for } \mathrm{i}<=\mathrm{t} \\
& \mathrm{hC}_{2}(\mathrm{t})=\operatorname{sum}\left(\mathrm{pi} / \mathrm{pC} C_{2}\right) \log \left(\mathrm{pi} / \mathrm{pC}_{2}\right) \text { for } \mathrm{i}>\mathrm{t} \\
& \mathrm{PC}_{1}=\operatorname{sum} \mathrm{P}_{\mathrm{i}} \mathrm{i}<=\mathrm{t} \text { and } \mathrm{PC}_{2}=\operatorname{sum} \mathrm{P}_{\mathrm{i}} \mathrm{i}>\mathrm{t}
\end{aligned}
$$

$\mathrm{PC}_{1}+\mathrm{PC}_{2}=1$ because the histogram is normalized

$\mathrm{Pi}=\mathrm{n}_{\mathrm{i}} / \mathrm{N}$, where $\mathrm{n}_{\mathrm{i}}$ is the occurrence of the gray level " $\mathrm{i}$ " in the image. $n_{i}$ is the histogram $h(i)$.

\section{SEGMENTATION RESULTS}

Some of the typical results of segmentation are shown below in Fig. 2 and 3.

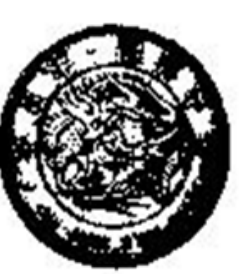

(d) MET

\section{CONCLUSIONS}

In this paper we discussed some of the traditional method of image segmentation with respect to numismatic coin data. The segmentation technique of the image could be used as per the required application or the usage as image is segmented on the basis of different features. The segmentation techniques are broadly categorized on the basis of detection of discontinuity and similarity of the image. Opting a single technique or method would not provide better optimized results. The result obtained using presented maximum entropy-based thresholding approach is quite promising. It can separate object from background image better than other approaches like Global Thresholding, Adaptive Thresholding.

\section{ACKNOWLEDGMENTS}

This work is financially supported by University Grant Commission (UGC), New Delhi, India through MRP.

\section{REFERENCES}

[1] N”olle, M., Penz, H., Rubik, M., Mayer, K., Holl"ander, I., Granec, R.: Dagobert-a new coin recognition and sorting system. In: Proceedings of the 7th International Conference on Digital Image Computing-Techniques and Applications (2003). .

[2] Van Der Maaten, L.J., Poon, P.: Coin-o-matic: A fast system for reliable coin classification. In: Proc. of the Muscle CIS Coin Competition Workshop, pp. 7-18 (2006).

[3] Reisert, M., Ronneberger, O., Burkhardt, H.: A fast and reliable coin recognition system. In: Hamprecht, F.A. 
Schn“orr, C., J“ahne, B. (eds.) DAGM 2007. LNCS, vol. 4713, pp. 415-424. Springer, Heidelberg (2007).

[4] Zambanini, S., Kampel, M.: Robust automatic segmentation of ancient coins. In: Proc. Conf. on Comp. Vision Theory and Appl., vol. 2, pp. 273-276 (2009).

[5] Image Segmentation Techniques Rajeshwar Dass, priyanka, Swapna Devi IJECT Vol. 3, Issue 1, Jan. $\operatorname{March}(2012)$.
[6] Segmentation Techniques For Image Analysis IJAERS/Vol. I/ Issue II/January-March, 2012.

[7] P.Daniel Ratna Raju and G.Neelima "Image Segmentation by using Histogram Thresholding" IJCSET January 2012 Vol 2, Issue 1,776-779. 